\title{
Teoria dos valores extremos aplicada na análise da Radiação Ultravioleta na cidade de Natal-RN
}

\author{
Alexandre Boleira Lopo, Washington L. F. Correia Filho, Maria Helena C. \\ Spyrides, Paulo Sérgio Lucio \\ Universidade Federal do Rio Grande do Norte (UFRN)-Programa de Pós-graduação em Ciências \\ Climáticas (PPGCC) \\ E-mail: alexandrelopo@hotmail.com
}

\author{
Javier Sigró, \\ Universidade Rovira i Virgili (Tarragona, Spain) \\ E-mail: javier.sigro@urv.cat
}

\begin{abstract}
Resumo: Através da teoria de valores extremos realizou-se uma análise de eventos de elevada intensidade de Radiação Ultravioleta (RUV) na cidade de Natal. A capital do Rio Grande do Norte é denominada pelos seus habitantes de "Cidade do Sol" em razão de sua elevada luminosidade e insolação, contudo esta cidade possui taxas de câncer de pele não melanoma acima da média das capitais do Nordeste do Brasil. A metodologia consistiu do uso da Teoria dos Valores Extremos via distribuição generalizada de valores extremos $(G E V)$ e distribuição generalizada de Pareto (GPD) para estimar os níveis e períodos de retorno do Índice UV. Os dados analisados são diários que correspondem às máximas do verão: 23 de dezembro a 23 de março (2001 a 2012). Os resultados mostraram tanto pela distribuição GEV como para GPD, que ocorrerá um extremo de RUV aproximadamente de 16 para os próximos cinco anos. Quanto ao parâmetro de forma (乡), a função de distribuição da GEV é uma Weibull negativa, enquanto a GPD é uma função de distribuição beta.
\end{abstract}

Palavras-chave: índice UV, Teoria dos Valores Extremos, níveis de retorno.

\section{INTRODUÇÃO}

Conforme o quinto relatório do IPCC (2013) há um cenário de mudanças climáticas em que o aquecimento global é inequívoco, nessa perspectiva o estudo dos extremos climáticos se torna cada vez mais relevante, além disso, os eventos raros ou extremos têm grande importância na climatologia, e suas estimativas probabilísticas são imprescindíveis para o planejamento e desenvolvimento das atividades sujeitas a efeitos adversos, especialmente estruturas de engenharia civil e agricultura.

Nesta investigação se estudou a Radiação Ultravioleta (RUV), visto que na Região Nordeste do Brasil (NEB), onde está localizado o Rio Grande do Norte (RN), e sua capital (local desta pesquisa), pesquisas indicaram projeções de aumento da UV e elevação do valor médio do Fluxo de Radiação Ultravioleta (FUV) no Hemisfério Sul, inclusive no NEB (latitudes de $-20^{\circ}$ a $\left.0^{\circ}\right)[10,17]$. Esta região apresenta uma climatologia da UVery (UV eritêmica ou biologicamente ativa) com valores considerados elevados [15]. Essa elevação do UV está associada à queda do ozônio total [17, 22], que no momento encontra-se em recuperação como resultado do Protocolo de Montreal, em vigor desde 1989 [25].

Natal é uma cidade turística de belas praias com elevada luminosidade solar (insolação e radiação solar) [3], sendo chamada pelos seus habitantes de "Cidade do Sol" [8]. Estas características podem representar um aumento no número de casos de câncer de pele não melanoma (CPNM) nesta cidade, que inclusive apresenta taxas de CPNM acima da média regional em relação às demais capitais do NEB [4].

$\mathrm{O}$ estudo baseado na teoria de valores extremos tem crescido nas últimas décadas, com diversas aplicações em várias áreas, incluindo-se as ciências da natureza. A maioria da teoria estatística se concentra em analisar o centro das distribuições, dando pouca importância para as 
caudas, que em vários casos possui um comportamento diferente da maioria das caudas das distribuições tradicionais, como a Normal e Gama.

O objetivo deste artigo é analisar o ajuste de diferentes distribuições de extremos às séries de índice UV do período de verão na cidade de Natal-RN.

\section{MATERIAIS E MÉTODOS}

\subsection{LOCAL DA PESQUISA}

Natal $\left(5^{\circ} 48^{\prime} \mathrm{Sul}\right.$ e $35^{\circ} 12^{\prime}$ Oeste) é uma cidade litorânea do Atlântico Sul, localizada na costa leste do NEB, que possui 2.968,4 horas anuais de brilho solar (insolação total) [3], com uma trajetória do Sol que varia com inclinação de $65^{\circ}$ a $90^{\circ}$ [1] e radiação solar global horizontal de média anual próxima de $5,0 \mathrm{kWhm}^{-2}$ [19]. A cidade possui clima tropical nordeste oriental, com temperatura do ar quente (média $\geq 18^{\circ} \mathrm{C}$ em todos os meses do ano) e semiúmida ( 4 a 5 meses $\operatorname{secos})[2]$.

O índice UV apresenta média anual igual a 11, classificado como "extremo" pela WMO, sendo que sete meses do ano e aproximadamente $80 \%$ dos dias nos meses de verão atingem este índice [15]. O índice UV, considerando o período anual, atinge às $8 \mathrm{~h}$ (horário local), índices categorizados pela WMO de risco "moderado" e "alto". No horário das $9 \mathrm{~h}$ alcança risco "alto" e "muito alto". O índice atinge valores de risco "muito alto" e "extremo" entre 10 e $12 \mathrm{~h}$, com predominância de risco "extremo" no intervalo 11 - $12 \mathrm{~h}$. A partir das $13 \mathrm{~h}$ os índices diminuem para risco "muito alto" e "alto", às $14 \mathrm{~h}$ para "alto" e "moderado" e às $15 \mathrm{~h}$ para "baixo" até o fim da tarde [15].

\subsection{IRRADIÂNCIA ERITÊMICA E ÍNDICE UV}

A irradiância eritêmica é dada pela convolução entre a irradiância solar espectral da UV que atinge a pele e o espectro de referência de ação eritêmica (espectro biológico de ação) [18].

O índice UV consiste de um fator de conversão da irradiância eritêmica e uma padronização numa escala numérica dividida em categorias associadas a cores (Tabela 1), que visa possibilitar uma rápida compreensão sobre a radiação ultravioleta biologicamente ativa. $\mathrm{O}$ índice UV foi formulado (Equação 1) pela Comissão Internacional de Iluminação (CIE- ISO 17166:1999 / CIE S007/E-1998) [24]:

$$
\text { índice } U V=\mathrm{k}_{\mathrm{er}} \int_{250 \mathrm{~nm}}^{400 \mathrm{~nm}} \mathrm{E}(\lambda) \cdot \mathrm{S}_{\mathrm{er}}(\lambda) \cdot \mathrm{d} \lambda
$$

Em que: $\lambda$ representa o comprimento de onda em $\mathrm{nm} ; \mathrm{E}(\lambda)$ a irradiância solar espectral em $\mathrm{Wm}^{-2} \mathrm{~nm}^{-1} ; \mathrm{S}_{\mathrm{er}}(\lambda)$ a referência do espectro de ação eritêmica; $\mathrm{d} \lambda$ o intervalo de comprimento de onda utilizado na integral e; $\mathrm{k}_{\text {er }}$ uma constante igual a $40 \mathrm{~m}^{2} \mathrm{~W}^{-1}$. O índice UV igual a 1 representa $0,025 \mathrm{Wm}^{-2}$, indicando que cada ponto na escala é equivalente a $25 \mathrm{~mW}$ por metro quadrado de UV [5].

O índice UV diário é um número inteiro e adimensional que representa o máximo valor diário numa superfície horizontal [24].

Tabela 1- Relação entre a faixa do índice UV e a categoria de risco para as pessoas.

\begin{tabular}{l|c|c}
\hline Categoria de risco & Faixa do índice UV & Cor \\
\hline Baixa & $0-2$ & Verde \\
Moderado & $3-5$ & Amarelo \\
Alto & $6-7$ & Laranja \\
Muito alto & $8-10$ & Vermelho \\
Extremo & $\geq 11$ & Violeta \\
\hline
\end{tabular}

Fonte: [24]. 


\subsection{DADOS DE ÍNDICE UV}

A investigação analisou as séries históricas das observações diárias do índice UV no período de 2001 a 2012, medidas pelo radiômetro multiespectral Ground-based Ultraviolet Radiometer (GUV), modelo 511-C instalado a altitude de $58 \mathrm{~m}$ no Laboratório de Variáveis Ambientais Tropicais do Centro Regional do Nordeste do Instituto Nacional de Pesquisas Espaciais (INPE/CRN/LAVAT) localizado em Natal, sendo transferido para o interior do RN, limitando ao período de 2001 a 2009. Os dados do índice UV do período de 2010 a 2012 foram obtidos na Estação Meteorológica (Modelo Davis com Sensor UV-6490) do Laboratório de Máquinas Hidráulicas e Energia Solar do Centro de Tecnologia da Universidade Federal do Rio Grande do Norte (UFRN/LMHES) na altitude de $60 \mathrm{~m}$. Os dados foram coletados no intervalo de 11 - 13h e estão entre 0 a 16 com $5 \%$ de incerteza. Os dados foram filtrados utilizando-se o período do verão ( 23 de dezembro a 23 de março).

\subsection{TEORIA DE VALORES EXTREMOS}

A teoria de valores extremos é fundamental para a modelagem de eventos raros ou extremos presentes na climatologia. Os fundamentos desta teoria foram desenvolvidos por Fisher-Tippett (1928), que definiram os três tipos possíveis de distribuições assintóticas de valores extremos, conhecidas como de Gumbel (tipo I), Fréchet (tipo II) e Weibull (tipo III) (Gumbel, 1958), estes casos especiais estão simplificados visto na Equação 1, conhecida como Distribuição Generalizada de Valores Extremos (GEV) [9, 12, 23].

Seja $\mu$ um dado limiar suficientemente grande, a GEV possui a função de distribuição acumulada dada por:

$$
F(x)=\exp \left\{-\left[1+\xi\left(\frac{x-\mu}{\sigma}\right)\right]^{-\frac{1}{\xi}}\right\}
$$

Quando $-\infty<x<\mu-\sigma / \xi$ para $\xi<0,-\infty<x<+\infty$ para $\xi$ tendendo a zero, $\mu-\sigma / \xi<x<$ $+\infty$ para $\xi>0$, sendo $\mu, \sigma$ e $\xi$, respectivamente, os parâmetros de localização, escala e de forma com $\sigma>0$. As distribuições de valores extremos de Fréchet e de Weibull correspondem aos casos particulares em que $\xi>0$ e $\xi<0$, respectivamente $[9,12,23]$.

Pickands (1975) mostrou que se $X$ é uma variável aleatória com função distribuição acumulada $\mathrm{F}(\mathrm{x})$, então, sobre certas condições, $F(x \mid u)=P(X \leq u+x \mid X>u)$ pode ser aproximada por uma Distribuição Generalizada de Pareto (GPD) $[6,13,20]$ :

$$
G(x \mid \xi, \sigma, u)=\left\{\begin{array}{c}
1-\left(1-\xi \frac{x-u}{\sigma}\right), \text { se } \xi \neq 0 \\
1-\exp \{-(x-u) \mid \sigma\}, \text { se } \xi=0
\end{array}\right.
$$

Em que $\sigma<0$ e $\xi$ são os parâmetros de escala e forma. A Equação (2) é valida para $\mathrm{x}-\mathrm{u}>0$, para $\xi>0$ e $0 \leq \mathrm{x}-\mathrm{u} \leq-\sigma / \xi$ se $\xi<0$.

Quando o modelo de GPD tem $\xi>0$ (modelo de cauda pesada), os extremos são mais frequentes do que quando o modelo tem $\xi<0$ ou $\xi=0$ (modelos de cauda leve) $[6,13,20]$.

No presente trabalho, $\mathrm{X}$ é a sequência de valores extremos de índice UV que excede um dado limiar, para uma estação individual, com 111,25 valores para cada ano, este 0,25 representa a contagem do ano bissexto.

A determinação dos extremos de RUV foi determinada da seguinte maneira: pela distribuição GEV, o extremo de RUV consiste de máximos trimestrais (dezembro a março); enquanto para a distribuição GPD, estes extremos são valores superiores ao percentil 95 (>95p), com os cálculos e testes foram realizados no software R [21].

\section{RESULTADOS}

A Tabela 1 apresenta os valores dos parâmetros das distribuições GEV e GPD estimados a partir das observações do índice RUV, e constata-se pelo parâmetro de localização 
(média dos valores para extremos) que ambas as distribuições possuem valor aproximadamente de 15, o que difere este valor seria o parâmetro de escala (desvio padrão para extremos) que é maior na distribuição GEV, devido à quantidade de valores de máximos extraídos, um total de 12 , e se verifica pelo parâmetro de forma (distribuição da série) valores negativos, indicando que a distribuição GEV se ajusta a distribuição de Weibull negativa, enquanto a GPD apresenta uma distribuição beta.

Tabela 1. Parâmetros distribuições GEV e GPD estimados para os índices UV.

\begin{tabular}{cccc} 
& \multicolumn{3}{c}{ Parâmetros das Distribuições } \\
\hline & localização & Escala & Forma \\
\hline GEV & 14,68 & 1,29 & $-1,06$ \\
GPD (95p) & 14,60 & 0,35 & $-0,13$ \\
\hline
\end{tabular}

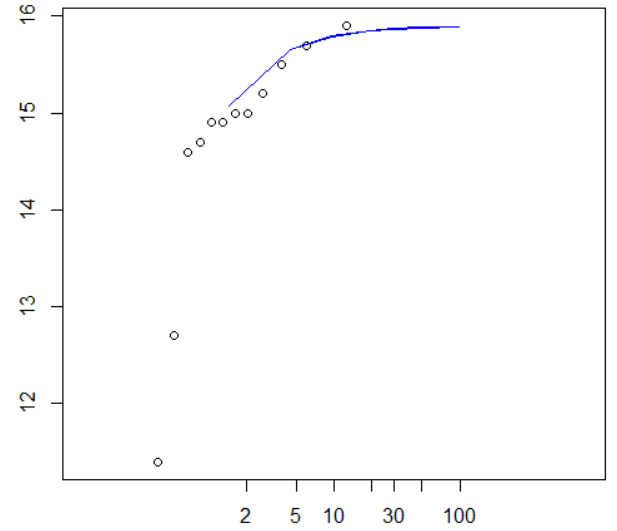

(a)

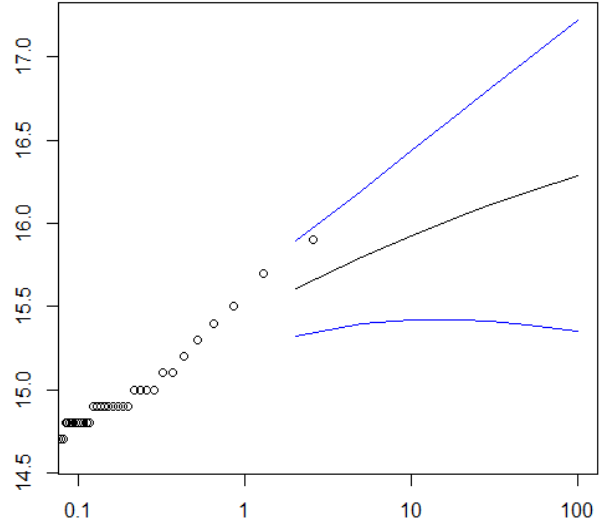

(b)

Figura 1 - Níveis e períodos de retorno para as distribuições GEV (à esquerda) e GPD (à direita) para o índice UV. O eixo-x representa o período de retorno (dado em anos), enquanto o eixo-y representa os níveis de retorno (índice RUV).

A Tabela 2 apresenta os níveis e períodos de retorno estimados para os índices RUV entre 2001 e 2012, e verifica-se que os valores em média são de aproximadamente 15. Verifica-se pela distribuição GEV, a estimativa para que ocorre um evento extremo de RUV de 15,65 nos próximos cinco anos, enquanto a estimativa observada pela distribuição GPD este valor extremo foi de 15,80. Constata-se que os valores tendem a se estabilizar após as estimativas destes extremos para períodos de retorno maiores que 10 anos, devido à forma da distribuição, já que o parâmetro de forma foi negativo em ambas as distribuições, o que resulta em uma estabilização nos níveis de retorno com o aumento do período de retorno.

Tabela 2. Períodos (em anos) e níveis de retornos estimados para os índices UV.

\begin{tabular}{cccccc}
\hline Período de retorno & $\mathrm{T}=0$ & $\mathrm{~T}=5$ & $\mathrm{~T}=10$ & $\mathrm{~T}=20$ & $\mathrm{~T}=30$ \\
GEV & 14,68 & 15,65 & 15,79 & 15,85 & 15,86 \\
GPD & 14,60 & 15,80 & 15,93 & 16,07 & 16,11 \\
\hline
\end{tabular}

\section{CONSIDERAÇÕES FINAIS}

O índice UV em Natal-RN varia predominantemente de 10 a 13 no período de verão, entre $11 \mathrm{~h}$ e $13 \mathrm{~h}$. As conclusões iniciais constaram que os parâmetros de localização para ambas as distribuições indicaram valores próximos a 15. Quanto aos níveis de retorno, as distribuições GEV e GPD indicaram que as estimativas do índice UV para os cinco anos são 
aproximadamente aos níveis de 16 . O parâmetro de forma $(\xi)$ indicou que a função distribuição acumulada para GEV é a Weibull negativa e para a GPD é a beta.

\section{AGRADECIMENTOS}

O presente trabalho foi realizado com apoio da Universidade Federal do Rio Grande do Norte através do Programa de Pós-Graduação em Ciências Climáticas (PPGCC/CCET) e das Instituições mantenedoras do autor: Universidade do Estado da Bahia e Instituto Federal de Educação Tecnológica da Bahia.

Os autores agradecem a Prof ${ }^{a}$ Neusa Paes Leme, Eng ${ }^{\circ}$ Francisco Raimundo da Silva (INPE/CRN) e ao Prof. Luiz Guilherme M. de Souza (UFRN/LMHES) pelas orientações e gentileza ao fornecer os dados.

\section{REFERÊNCIAS}

[1] ARAÚJO, V. M. D. Parâmetros de conforto térmico para usuários de edificações escolares no litoral nordestino brasileiro. Tese de doutorado. USP. São Paulo, 1996.

[2] BRASIL, IBGE. Mapa de Clima do Brasil. [Internet], 2013. Disponível em ftp://geoftp.ibge.gov.br/mapas_tematicos/mapas_murais/. Acesso: Novembro-2013

[3] BRASIL, INMET. Normais Climatológicas do Brasil 1961-1990. Brasília, DF, 2009.

[4] BRASIL, INCA-Instituto Nacional de Câncer. Estimativa 2012-Incidência de Câncer no Brasil. Ministério da Saúde do Brasil, 2011.

[5] CORRÊA, M. P. Índice ultravioleta: avaliações e aplicações. Tese (Doutorado Ciênciasmeteorologia) Instituto de Astronomia, Geofísica e Ciências Atmosféricas, USP, São Paulo, 2003.

[6] EMBRECHTS, P..; KÜPPELBERG, C.; MIKOSCH,T. Modelling extremal events for insurance and finance, New York: Springer.1997.

[7] FISHER, R. A.; TIPPETT, L.H.C. Limiting forms of the frequency distribution of the largest or smallest number of the sample. Proc. Cambridge Philos. Soc., v. 4, 1928.

[8] FURTADO, E. M. A onda do turismo na cidade do sol: a reconfiguração urbana de Natal, 2005.

[9] JENKINSON, A. F. The frequency distribution of the annual maximum (or minimum) values of meteorological elements. Q. J. R. Met. Soc., v. 81, 1955.

[10] HERMAN J.R. Global increase in UV irradiance during the past 30 years (1979-2008) estimated from satellite data. Journal of Geophysical Research, vol. 115, p. 01-15, 2010.

[11] IPCC, Intergovernmental Panel on Climate Change, Task Group ons Scenarios for Climate Impact Assessment, 2013.

[12] KATZ, R. W.; PARLANGE, M. B.; NAVEAU, P. Statistics of extremes in hydrology. Advances in Water Resources, v. 25, p. 1287-1304., 2002. 
[13] KUNKEL, K.E., PRISTKE, R.A., CHANGNON, S.A.,Temporal fluctuation in weather and climate extremes that cause economic and human health impacts-a review. Bulletin of American Meteorological Society, 80, 1077-1098, 1999.

[14] LEE-TAYLOR, J., MADRONICH, S. Climatology of UV-A, UV-B, and Erythemal Radiation at the Earth's Surface, 1979-2000, National Center for Atmospheric Research Boulder, Colorado, 2007.

[15] LOPO, A. B. et al. Radiação ultravioleta, ozônio total e aerossóis na cidade de NatalRN. HOLOS, v. 6, p. 3-21, 2013.

[16] MCKENZIE, R.L. et al. Changes in biologically active ultraviolet radiation reaching the Earth's surface. Photochem Photobiol. Sci., v. 2, p. 1-4, 2003.

[17] MCKENZIE et al. Ozone depletion and climate change: impacts on UV radiation. Photochem. Photobiol. Sci., 10, 182-198, 2011.

[18] MCKINLAY, A., F. DIFFEY, B. L. A reference action spectrum for ultraviolet-induced erythema in human skin. In: Passchler and Bosnajokovic ed. Human exposure to ultraviolet radiation: Risks and regulations. Amsterdã, Elsevier, 1987.

[19] PEREIRA, E. B. et al. Atlas brasileiro de energia solar, INPE, São José dos Campos, Brasil. ISBN 978-85-17-00030-0, 2006.

[20] PICKANDS III, J. Statistical inference using extreme order statistics. The Annals of Statistics, p. 119-131, 1975.

[21] R DEVELOPMENT CORE TEAM. R: A language and environment for statistical computing. 2011, Vienna, Austria: R Foundation for Statistical Computing. ISBN 3-900051-07-0.

[22] SAHAI, Y., KIRCHHOFF, V., LEME, N. M. Total ozone trends in the tropics. Journal of Geophysical Research, v.105, n.D15, p. 19823-19823. EUA, 2000.

[23] SANSIGOLO C. A. Distribuições de extremos de precipitação diária, temperatura máxima e mínima e velocidade do vento em Piracicaba, SP (1917-2006). Revista Brasileira de Meteorologia, v.23, n.3, 341-346, 2008..

[24] WHO. Global Solar Indice UV: A Practical Guide. World Health Organization, World Meteorological Organization (WMO), United Nations Environment Program (UNEP), and International Commission on Non-Ionising Radiation Protection (ICNRP), 18 p. Geneva, 2002.

[25] WMO. Scientific assessment of ozone depletion, 2010. World Meteorological Organization, Report No. 47. Geneva, 2011. 